Revista de

Contabilidade e

Organizações

www.rco.usp.br
DOI: http://dx.doi.org/10.11606/rco.v9i25.86853
Journal of

Accounting and

Organizations

www.rco.usp.br

\title{
O processo orçamentário e a tomada de decisão de gestores em um hospital público
}

Gabryel Lopes Sola ${ }^{a}$; Carlos Alberto Grespan Bonacim ${ }^{a}$

a Universidade de São Paulo

Informações do Artigo

Histórico do Artigo

Recebido: 02 de novembro de 2014

Aceito: 30 de julho de 2015

Palavras-chave:

Processo orçamentário.

Hospital público.

Tomada de decisão.

Gestor clínico.

\begin{abstract}
Resumo
Ao deparar-se com um hospital público em que há a presença da ferramenta orçamentária e que conta com ausência de incentivos financeiros para o alcance de metas orçamentárias, com a complexidade da gestão, incerteza da tarefa e do embate entre profissionais da área médica e da administração, há o interesse do entendimento de como é a utilização dessa ferramenta para a tomada de decisão. Portanto, este trabalho aplicou um estudo de caso, utilizando entrevistas semiestruturadas, no Hospital das Clínicas de Ribeirão Preto para esse entendimento. Os resultados demonstram que a interação dos gestores com o orçamento se dá predominantemente via planejamento de materiais e incorporação de novas tecnologias. Além disso, o instrumento de gestão pode impactar as escolhas intertemporais do gestor clínico. Por fim, os principais critérios de tomada de decisão observados e que estão presentes no processo orçamentário são: custo do material, acompanhamento do consumo de recursos, impacto no protocolo clínico, investimento em atualização tecnológica e normatização.
\end{abstract}

Copyright (C) 2015 FEA-RP/USP. Todos os direitos reservados

\section{INTRODUÇÃO}

Esta pesquisa aplicou um estudo de caso, tendo como unidade de análise o Hospital das Clínicas de Ribeirão Preto, para observar como o gestor clínico utiliza o processo orçamentário dessa instituição para tomada decisão, considerando a possível presença de conflitos entre gestão e atividade médica, e as particularidades de um hospital público.

Esses hospitais têm sido observados pela literatura como instituições complexas que lidam com a vida do ser humano (GLOUBERMAN; MINTZBERG, 1996). No entanto, qual é o valor limite gasto para salvar uma vida? Essa decisão está diante dos gestores dos hospitais, os quais trabalham com orçamentos em que recursos são escassos.

Essa complexidade se dá pela dificuldade de medir a qualidade do serviço prestado devido à sua intangibilidade, aos métodos orçamentários não espelharem essa qualidade, à dificuldade em estabelecer procedimentos para uma tarefa incerta, tendo que confiar na cultura da instituição, na produção e no consumo do serviço, com ocorrência simultânea e aparente dissociação entre área médica e área administrativa (PETTERSEN, 1995; PETTERSEN; NYLAND, 2006).

Os hospitais consomem quase a metade das despesas destinadas ao setor da saúde (ROTTA, 2004), no entanto, não é possível estabelecer um padrão de financiamento destes, uma vez que são financiados por diversas fontes de receitas, diversos convênios e contratos com o Sistema Único de Saúde (SUS), receitas financeiras, entre outros (BITTAR; MAGALHÃES, 2010).

Os hospitais conveniados com o SUS são remunerados considerando uma tabela de pagamento préestabelecida, no entanto, há evidências que mostram que esses valores são menores que o custo real quando se trata de procedimentos de baixa complexidade, podendo chegar a $30 \%$ do custo mas, para alguns procedimentos de alta complexidade (transplantes e cirurgias cardíacas, por exemplo) as evidências apontam remuneração superior ao

Autor Correspondente: Tel (16) 36023000

E-mail : gabryelsola@gmail.com (G. L. Sola); carlosbonacim@yahoo.com.br (C. A. G. Bonacim)

FEARP - USP - Avenida dos Bandeirantes nº 3900 - Sala 5 - Bloco A - Monte Alegre - 14040900 - Ribeirão Preto, SP - Brasil 
custo (LA FORGIA; COUTTOLENC, 2009). Sendo assim, a complexidade de uma entidade hospitalar, somada a fontes de recursos que podem não cobrir o gasto efetivo de determinados procedimentos, faz com que a gestão desses hospitais se torne um desafio, uma vez que, teoricamente, exige elevada eficiência quanto à gestão dos recursos (BONACIM; ARAUJO, 2010).

Os hospitais universitários são considerados financeiramente debilitados, por serem mais dispendiosos, devido à adição de atividades de ensino e pesquisa, pelo atendimento de alta complexidade e por serem referenciados em tecnologia de ponta (COSTA; SILVA; TIBÉRIO, 2003; DALLORA; FORSTER, 2008). Essa debilitação deveria fazer com que houvesse um aumento de recursos da iniciativa privada, no entanto, o elevado custo dessa atividade inibe o incentivo a essa iniciativa. Portanto, a tendência é que esse tipo de atividade continue sendo custeada pelo poder público para a maioria dos serviços (MÉDICI, 2001).

Outra saída seria a adoção de ferramentas de gestão, porém, a gestão em saúde enfrenta as dificuldades em utilizar os modelos de gestão aplicados à indústria devido à sua especificidade, ao aspecto intangível da prestação de serviços, à simultaneidade de prestação e de consumo do serviço pelo paciente (cliente) e na dificuldade da padronização de procedimentos que aumenta quanto maior for a característica intangível do serviço prestado (DAL POZ; PIERANTONI; VARELLA, 1997; ROTTA, 2004; FADEL; FILHO, 2009).

É possível encontrar hospitais públicos gerenciados por meio de ferramentas de gestão como orçamentos. Essa ferramenta refere-se a um plano que a organização usa para obter e consumir recursos financeiros e não financeiros durante um período de tempo (LU, 2011). O orçamento pode ser usado como um mecanismo de gestão, como forma de autorização a gestores para que gastem determinada quantidade de recursos, como forma de planejamento e controle, como uma ferramenta para influenciar o comportamento do gestor e motivar financeiramente suas práticas de tomada de decisão, como forma de julgamento do desempenho do gestor e cálculo de remuneração (MACINATI, 2010). Sendo assim, essa ferramenta poderia contribuir com a melhoria da gestão, desde que incorporadas as características específicas da atividade.

Ainda na gestão hospitalar, há evidências da caracterização da separação entre as atividades de gestão e atividades médicas (LAPSLEY, 2001). Por um lado há a discussão sobre a liberdade média e por outro a limitação de recursos da entidade. Enquanto no primeiro a decisão da alocação dos recursos ficaria com o próprio médico, no segundo há a escassez de recursos devido à extensão dessa decisão para pacientes com quadros clínicos semelhantes no presente e no futuro. (PRESTON; COOPER; COOMBS, 1992).

As pressões orçamentárias, com o objetivo de nortear a prática médica para que o médico não renuncie à eficiência, seriam transmitidas pela hierarquia, acompanhada de sanções caso houvesse o descumprimento, como um possível meio de forçá-los a priorizar ou repensar suas prioridades entre a saúde e a rentabilidade da organização (GEORGESCU; NARO, 2012).

Acrescenta-se a esses conflitos entre as atividades de gestão e médicas a influência das escolhas intertemporais do gestor, pois a ferramenta orçamentária com excessivo foco no curto prazo pode desencorajar ideias novas, cujas despesas incorrem no longo prazo (MERCHANT, 1990; LIEBERMANN; UNGAR, 2002).

Alinhando a especificidade da fonte de recursos de cada hospital público, como apontado por Bittar e Magalhães (2010), com o processo orçamentário utilizado, somado a isso os desafios da gestão, e suas escolhas que envolvem o bem-estar da vida humana e a finitude da vida, e considerando, também, as particularidades do sistema de remuneração de um hospital público, este trabalho empregou um estudo de caso ao Hospital das Clínicas da Faculdade de Medicina de Ribeirão Preto da Universidade de São Paulo (doravante HCRP), utilizando entrevistas semiestruturadas, e verificou como é utilizado o processo orçamentário para tomada de decisão dos gestores clínicos, intermediários desse conflito entre as lógicas administrativa e médica.

Este estudo tem seu foco voltado para gestores clínicos que, conforme apontado pela literatura (NYLAND; PETTERSEN, 2004), teriam tensões entre o cumprimento de metas orçamentárias e o atendimento das necessidades do corpo clínico da instituição, ou seja, teriam o papel de intermediadores desse conflito.

O objetivo geral da pesquisa é verificar como o processo orçamentário da instituição é utilizado para tomada de decisão dos gestores clínicos, tendo como objetivos específicos: verificar a participação dos gestores clínicos na elaboração do orçamento; verificar a utilização do orçamento para tomada de decisão; e verificar variáveis comuns presentes na tomada de decisão do gestor clínico e no processo orçamentário.

O diferencial deste trabalho é que são encontrados poucos trabalhos que discutem a relação do orçamento com a tomada de decisão em um hospital público. Além disso, espera-se obter como resposta o levantamento das variáveis consideradas no processo de tomada de decisão do gestor clínico e quais são passíveis de serem encontradas no processo orçamentário, demonstrando como é a sua utilização. Com essas variáveis, objetiva-se contribuir com 
pesquisas por meio da sistematização de instrumento de coleta de dados para investigar a gestão de Hospitais Públicos que possuem similaridade ao hospital estudado.

Esta pesquisa está estruturada, além do tópico de introdução, em: referencial teórico, para justificar as relações propostas neste trabalho; metodologia da pesquisa, que descreve a forma com que se conduziu este estudo; apresentação dos resultados, com os achados aplicando a metodologia no hospital selecionado; e, por fim, as considerações e relações dos achados com os objetivos propostos.

\section{REFERENCIAL TEÓRICO}

\subsection{Ferramenta Orçamentária}

Os gestores utilizariam o orçamento para especificar os objetivos e planos da organização como um todo e assim alocando os recursos limitados da entidade econômica. Contudo, o orçamento também pode servir como proposta de estruturar a alocação de esforços de diferentes departamentos e divisões, especificando um plano de metas e comunicando os objetivos organizacionais. É comum a utilização de alvos com a proposta de motivar os gestores e os empregados a alcançar os objetivos de seu departamento (ETHERINGTON; TJOSVOLD, 1998).

O alcance das metas (ou alvos) pode ser utilizado como base de avaliação de desempenho do gestor atribuindo a ele a responsabilidade de resultados e, também, como base de sua remuneração. Sendo assim, o orçamento é frequentemente utilizado para facilitar a avaliação do comprometimento do gestor de nível intermediário (na hierarquia) às prioridades da organização (ABERNETHY; BROWNELL, 1999). No entanto, como essas metas não necessariamente ficam para o nível intermediário de gestão, elas podem variar conforme o cargo e o seu tipo de decisão: estratégica, tática, e operacional.

Já o orçamento público, semelhante ao orçamento privado, tem como um de seus objetivos a tentativa de coordenar e balancear as preferências políticas e os bens públicos contra a limitada disponibilidade de recursos, ou seja, contra os recursos escassos (KINNER; PELLEGRINI, 2009). No entanto, não é comum observar a remuneração atrelada ao orçamento da entidade pública.

O Ministério da Saúde atende aos requisitos legais e constitucionais, sendo assim, elabora o Plano Plurianual (PPA), Lei de Diretrizes Orçamentárias (LDO) e a Lei Orçamentária Anual (LOA). O PPA contém as diretrizes, objetivos e metas para um período de quatro anos. A LDO orienta a elaboração da LOA, e esta última discrimina a receita e a despesa que deverão atender aos princípios da anualidade, unidade e universalidade. Sendo assim, a União, o Distrito Federal, os Estados e os Municípios deverão se basear em seus planos de saúde para a elaboração das propostas orçamentárias.

Portanto, a unidade de análise desta pesquisa está vinculada ao PPA e à LOA da Secretaria Estadual da Saúde do Estado de São Paulo. Isso significa, portanto, que essa instituição deve elaborar seu orçamento quadrienal e anual considerando o tipo de gasto coberto por estas leis.

Merchant (1985) apresentou evidências de que a participação dos gestores em reuniões que discutem o processo orçamentário e metas vinculadas a ele, diminui a propensão de criar folga nesse processo. Este termo, "folga orçamentária", foi definido pelo autor como o excesso de valores orçados em uma área em face àquilo que é realmente necessário. Além disso, o autor também evidenciou que a capacidade de monitoramento também pode reduzir a propensão do gestor em criar folga.

A aceitação do orçamento pelo gestor dependerá de sua tolerância à ambiguidade, que pode aceitar ou não as incertezas inerentes à tarefa e ao ambiente embutidas ao orçamento (HARTMANN, 2005).

Na saúde, em muitos casos, a vida do paciente fica dependente da qualificação profissional do médico. E, por meio do seu treinamento profissional, ele tem que ser capaz de tratar pacientes com multitraumas sem tempo hábil para consulta ao manual de procedimentos ou às regras formais da entidade, ou seja, os gestores administrativos, por sua vez, não influenciam diretamente no que ocorre nessa interação (PETTERSEN, 1995).

Considerando essas características específicas da atividade hospitalar, é possível fazer inferência em relação às consequências para o processo orçamentário. Combinando a interação intensiva de médicos e pacientes com o alto grau de ambiguidade do desempenho, devido à sua intangibilidade, os mecanismos de controle 
orçamentários ficam reféns da confiança no aspecto cultural da entidade (PETTERSEN, 1995).

Esse controle cultural implica em padronização das normas e valores por meio de treinamento profissional (OUCHI, 1979). Sendo assim, devido a essa intangibilidade, considerada característica do atendimento hospitalar, essas entidades buscam aperfeiçoar a cultura hospitalar e transmiti-la aos médicos por meio de treinamentos. Essa padronização de normas e valores pode influenciar o processo orçamentário da entidade.

Esse processo, enquanto mecanismo de controle da gestão, também encontra resistência quando não há uma identificação do profissional médico com os objetivos gerenciais especificados e seus valores, e isso implica na não obtenção dos benefícios de se ter este tipo de controle (ABERNETHY; STOELWINDER, 1991). Essas organizações podem ser caracterizadas, portanto, com a presença de uma separação entre a estrutura administrativa burocrática e os profissionais dessa estrutura, geralmente médicos (LAPSLEY, 2001). E, na literatura, é possível encontrar pesquisas (COOMBS, 1987; PRESTON; COOPER; COOMBS, 1992; LAPSLEY, 2001)

Preston, Cooper e Coombs (1992) relatam debates em que o princípio da liberdade médica foi discutido. Segundo os autores, é esperado que o médico resista ao controle orçamentário em nome dessa liberdade. No entanto, esse mesmo estudo também aponta debates que discutem que a tecnologia usada ao paciente (juntamente com os recursos empregados para o tratamento de sua doença) teria que ser estendida aos demais pacientes que ainda não apresentam tal enfermidade, incorrendo em aumento no consumo de recursos na incorporação de tecnologias em saúde.

Historicamente, médicos têm reivindicado que as decisões médicas deveriam ser baseadas principalmente em objetivos e padrões profissionais e não de acordo com qualquer lógica econômica ou plano administrativo (HOPWOOD, 1992; NYLINDER, 2011). Pode-se acrescentar que as decisões podem ter correspondência com o valor da vida que é inestimável, e que podem não encontrar respaldo em orçamentos cujos limites de gastos já estão definidos.

Nyland e Pettersen (2004) estudaram a relação de informações contábeis e orçamentárias com a tomada de decisão de gestores e médicos de um hospital universitário norueguês e encontraram que, em muitos aspectos, os gestores dos departamentos clínicos são os mediadores de dois diferentes mundos de decisões clínicas e de racionalidade econômica. Além desses conflitos, há argumentos que demonstram que a atividade médica não é facilmente adaptável aos controles administrativos burocráticos, como o orçamento, pois há genuínas dificuldades em relacionar os inputs e os outputs na prática médica (COOMBS, 1987). No entanto, essa transformação das atividades terapêuticas em equivalentes de caixa e a mudança para a orientação financeira têm mudado a forma de argumentação em organizações de saúde (KURUNMAKI, 1999).

A necessidade médica em relação à gravidade de doenças e a necessidade de serviços para aliviar essas doenças são critérios considerados importantes para o tomador decisão em alocar os recursos escassos da saúde. Se o orçamento é fixo, o custo de oportunidade de adicionar uma nova tecnologia consiste na redução de recursos para outros serviços devido à realocação desses recursos (escassos) para as novas tecnologias (ROGOWSKI et al., 2014).

Portanto, percebe-se que o gestor em saúde tem, por um lado, a decisão baseada no orçamento e, por outro lado, a de incorporar novas tecnologias. No entanto, como esses recursos são escassos haverá a realocação, cabendo ao gestor decidir qual é a decisão que aumentará os benefícios aos clientes (pacientes).

\subsection{Orçamento sob a Ótica das Escolhas Intertemporais}

Problemas de escolhas intertemporais são inerentes à prática de gestão (LAVERTY, 1996). Essas escolhas envolvem decisões como investimento em tecnologia, treinamento, entrada em novos mercados, entre outros. A questão que envolve essa escolha na gestão ocorre com relação à maximização de resultados ou ao atingimento de objetivos nos quais as decisões em que o caminho da ação que é melhor no curto prazo não é o mesmo caminho da ação que é melhor no longo prazo (LAVERTY, 1996).

De modo complementar, as escolhas intertemporais podem ser definidas como as decisões que envolvem tradeoff entre os custos e os benefícios que ocorrem diferentes no tempo (FREDERICK; LOEWENSTEIN; O'DONOGHUE, 2002).

A característica central desse tipo de escolha é o tradeoff entre presente e futuro. Ilustrando, pode ser a desistência de benefícios presentes para aumentar quantidade de futuros recebimentos, fazendo, assim, um investimento. Por outro lado, é o aumento de benefícios presentes em detrimento da diminuição de benefícios 
futuros. Indiferente ao tipo de relação entre o presente e o futuro isso pode ser resumido em uma taxa de desconto. Ao tomar decisão em uma situação de escolha intertemporal o indivíduo implicitamente determina essa taxa (LIEBERMANN; UNGAR, 2002).

Por um lado, segundo a literatura, tem-se a própria decisão do gestor que influencia o aspecto intertemporal e, por outro, se sugere que o próprio sistema de gestão da entidade é capaz de influenciar o gestor em sua tomada de decisão concentrada no curto prazo ou no longo prazo. Merchant (1990), por meio de um estudo de campo, fez entrevistas para descobrir a incidência de duas causas importantes de disfunções no sistema de controle: a manipulação de desempenho do curto prazo e a orientação ao curto prazo de gestores. Na investigação foi verificado que essa manipulação era relatada devido a pressões orçamentárias que eles enfrentavam. Portanto, as escolhas intertemporais do gestor podem ser influenciadas pelas ferramentas de gestão que a entidade utiliza.

O processo orçamentário pode produzir um gestor míope, com excessiva orientação no curto prazo e, com isso, desencorajar novas ideias, principalmente quando as despesas serão incorridas no longo prazo, ou quando há pagamentos incertos (MERCHANT, 1990). A orientação gerencial em curto prazo é definida como a extensão do foco dos gestores em suas decisões, na tentativa de alcançar o desempenho dentro do período orçamentário corrente, que é de um ano (VAN DER STEDE, 2000).

\section{METODOLOGIA DA PESQUISA}

A observação da utilização do processo orçamentário do HCRP para tomada de decisão de seus gestores clínicos se deu por meio da condução de um estudo de caso. A escolha desse hospital se deu por conveniência e se justifica por ser considerado um complexo institucional, devido a suas complexidades orçamentárias, com a presença de diversas fontes de recursos para atender o consumo de atividades heterogêneas (conforme descrito no capítulo três deste trabalho). Além disso, essa instituição possui uma equipe de trabalho de 5.773 servidores e oferece assistência à saúde de nível terciário para a região de Ribeirão Preto/SP composta por, aproximadamente, quatro milhões de pessoas, além de atender pacientes referenciados de outros Estados (HCRP, 2014).

O HCRP é uma autarquia pública estadual, criado em 1956, vinculado administrativamente à Secretaria da Saúde do Estado de São Paulo e associado à Universidade de São Paulo para fins de ensino, pesquisa e prestação de serviços médico-hospitalares. Integra o SUS desde 1988 e é caracterizado como hospital de grande porte, de alta complexidade e de referência terciária para a região nordeste de São Paulo.

As fontes de recursos desse hospital compreendem valores advindos das esferas federal (aproximadamente 25\%) e estadual (aproximadamente 70\%), além de contar com recursos da Clínica Civil (HCRP, 2014). Portanto, essa instituição mantém dois orçamentos, um para atender a Secretaria da Saúde do Estado de São Paulo e outro que engloba as demais fontes de recursos, para aplicá-los a despesas não cobertas pelo orçamento estadual.

Para os gestores do HCRP não necessariamente interessa saber a origem desses recursos, pois é realizada uma previsão anual de materiais de consumo dos centros de custos pertencentes a esses gestores. O hospital é dividido em 276 centros de custos ativos (em 2014), e cada centro de custo tem um responsável, podendo ser os gestores ou pessoas indicadas por ele.

Além desse recurso para consumo, os departamentos clínicos têm disponível um percentual da verba do Faturamento SUS (3\% após ajustes). Esse recurso é encaminhado aos departamentos proporcionalmente à sua produtividade, apontada pelo departamento que faz a Gestão de Convênios (GECON). O repasse dessa verba tem o objetivo de fazer com que se dê maior agilidade aos departamentos, em relação a consumo, e possibilita o planejamento de investimentos na área do gestor.

A primeira interação com a entidade se deu por meio de uma entrevista com a diretora da Assessoria Técnica, que se prontificou a contribuir com a pesquisa e forneceu informações sobre as demais pessoas-chave que poderiam também contribuir com as necessidades de informação deste trabalho.

Nessa interação indicou-se um funcionário que está responsável diretamente pela elaboração do orçamento de gastos supridos pela verba estadual, uma funcionária responsável pelos convênios com o SUS, diretora do Grupo Executivo de Convênios (GECON) e uma funcionária responsável pela gestão de indicadores da instituição, do Grupo de Avaliação de Desempenho (GAD).

Este trabalho utiliza o termo "gestor clínico" como sendo aquele responsável por unidades envolvidas com 
a atividade fim do hospital. Ressalta-se que essa é uma associação com a função exercida e não com a formação do gestor. Esse termo foi adaptado de Nyland e Pettersen (2004) que associa o departamento clínico à presença do tratamento de pacientes. Foi realizada, portanto, a seleção dos gestores clínicos juntamente com pessoas-chave do hospital: ao todo foram escolhidos 10 indivíduos, tais que atenderam à definição de gestor clínico proposta neste trabalho.

Essa seleção conta com justificava na literatura, pois os resultados encontrados por Nyland e Pettersen (2004) mostraram que os gestores clínicos intermediariam os conflitos entre as lógicas na instituição.

Além desses gestores foram incluídos seis gestores que são subordinados ao nível hierárquico a que se encontram os dez gestores clínicos anteriormente escolhidos. Destaca-se que esses outros seis também atendem à definição de gestor clínico, no entanto, ficam encarregados de subunidades da instituição. Três gestores são subordinados ao Departamento de Apoio Médico e três à Divisão de Enfermagem.

Os impedimentos quanto à obtenção desses dados estão minimizados devido à concordância, da alta gestão, quanto à realização deste projeto e pela aprovação pelo Comitê de Ética em Pesquisa da Faculdade de Filosofia Ciências e Letras de Ribeirão Preto-USP e do Hospital das Clínicas da Faculdade de Medicina de Ribeirão Preto da USP.

A coleta de dados foi realizada por meio de entrevistas semiestruturadas, que permitem aos entrevistados certo grau de liberdade para explicar seus pensamentos ou transmitir experiências, relacionados ao assunto questionado. Além disso, permitem que algumas respostas sejam exploradas com mais profundidade (MARGINSON, 2004).

O roteiro para entrevista foi baseado nos trabalhos de Pettersen (1995), Nyland e Pettersen (2004) e Pettersen e Nyland (2006). Esses trabalhos pesquisaram a relação do orçamento com os gestores de hospitais utilizando roteiros de entrevistas semiestruturadas. Tentou-se contato com esses autores, via e-mail, no entanto, não houve resposta. O roteiro não é descrito de forma integral nesses trabalhos, mas no corpo do texto dos artigos constam as questões utilizadas. A partir dessas questões foi elaborado um roteiro para a condução das entrevistas semiestruturadas.

Realizou-se uma análise de conteúdo, com foco na análise temática e de coocorrência. Esta técnica de investigação tem como finalidade obter uma descrição objetiva, sistemática e quantitativa do conteúdo de determinada comunicação. Para submeter entrevistas a esse tipo de análise elas devem se referir a um mesmo tema, terem sido obtidas por técnicas idênticas e serem realizadas por indivíduos semelhantes (BARDIN, 2011). O tema comum será o processo orçamentário, a técnica empregada será a mesma para todos os entrevistados e o indivíduo que coletará todos os dados será o próprio pesquisador deste trabalho, garantindo, assim, maior validade para este estudo de caso.

O software utilizado para análise de conteúdo será o Sphinx, que foi adquirido pela Faculdade de Economia, Administração e Contabilidade de Ribeirão Preto.

O banco de dados gerado a partir do software foi analisado quantitativamente em relação à frequência de aparição de elementos relacionados ao processo orçamentário da instituição. Além disso, será identificado qual é o contexto utilizado quando esses elementos aparecem.

Portanto, será realizada a análise temática dos discursos, observando a frequência dos temas extraídos do conjunto dos discursos. Conforme Bardin (2011) apresenta, a análise temática somente, apesar de indispensável, prejudica a real interpretação dos dados, sendo assim, foi realizada a análise das coocorrências na tentativa de identificar relações entre os elementos da mensagem.

\section{APRESENTAÇÃO E ANÁLISE DOS RESULTADOS}

Os resultados deste trabalho estão baseados nas entrevistas com pessoas-chave do hospital, nas entrevistas realizadas com os gestores clínicos selecionados, na análise documental e na observação referente à participação na reunião da CAPC. Trata-se da triangulação dos dados sugerida por Voss, Tsikriktsis e Frohlich (2002). 


\subsection{Características dos Gestores}

Antes da análise dos discursos captados pelo roteiro semiestruturado descrito na metodologia deste trabalho, que visa identificar a utilização do processo orçamentário, pelo gestor clínico, para tomada de decisão, foram identificadas as características principais dos gestores clínicos entrevistados.

Com relação à formação, foram identificados quem são os gestores clínicos médicos e quem tem outra formação. Dentre estes, nove gestores têm formação médica e seis gestores têm formação não médica (enfermagem, farmácia).

Foi observado que os gestores selecionados estão, em média, há mais de 20 anos na instituição e ocupam cargo de gestão, em média, há 10 anos. Somente quatro gestores ocupam a função há menos de cinco anos. No entanto, alguns ocupavam função de apoio gerencial antes de assumir cargo de gestão.

\subsection{Processo Orçamentário e o Gestor Clínico}

A análise da utilização do processo orçamentário pelo gestor se deu, primeiramente, pela análise da importância e do entendimento que ele tem sobre esse processo.

De forma geral, eles veem que esse processo visa à captação, ao planejamento de aplicação e ao controle de recursos, com vistas a manter a assistência do hospital, dimensionar a atividade e auxiliar no atingimento dos objetivos da instituição.

Há a interação com o orçamento ao elaborar a programação de materiais, cujos valores já estão predefinidos, ou seja, há um teto de gasto definido para o setor, no entanto, não há valores pecuniários motivando o gestor a cumprir os gastos planejados, ou até mesmo reduzi-los. A participação e a motivação são apontadas com relação direta com o esforço empregado para atingir as metas orçamentárias (SEARFOSS; MONCZKA, 1973), isso possivelmente reduziria custos de transação, conforme apontado pela literatura.

Quando discutido sobre o conhecimento do processo orçamentário da instituição, a maioria dos gestores clínicos (doze gestores) aponta conhecimento amplo desse processo, ou seja, do planejamento e de dados de forma geral, sem profundidade em relação à forma de elaboração, do total de recursos destinados à instituição, recursos destinados para as demais áreas do hospital, e resultado de monitoramentos. Dois deles citaram ter um bom conhecimento do orçamento e três gestores apontam desconhecer esse processo.

Esse conhecimento mais geral do processo se mostra devido à participação se dar predominantemente pelo planejamento de materiais do setor, confirmando os dados iniciais obtidos por meio de entrevistas com pessoas-chave.

Verifica-se, portanto, que a participação dos gestores na elaboração do orçamento da instituição é, para a maioria dos gestores, limitada ao planejamento de materiais do setor, que conta com a inclusão, alteração e exclusão de materiais, mantendo o teto de gastos já estipulado para o setor.

Um dos gestores apontou que ele toma maior conhecimento do orçamento quando há reuniões entre a administração e os gestores para discutir esse assunto. Sete gestores citaram que essas reuniões são esporádicas, por convocação, conforme a necessidade de se reduzir gastos.

Essa convocação, aparentemente, independe da hierarquia do setor, se considerar a informação dos gestores clínicos entrevistados.

Dois outros gestores contribuíram com esse ponto de vista, pelo qual há somente a exposição de números, sem tomada de decisão por parte dos gestores, as reuniões são expositivas em relação às necessidades da instituição, por exemplo, a de corte de gastos.

Percebe-se, portanto que a ordem é transmitida pela hierarquia, no entanto, há a preocupação em manter o nível e a quantidade de atendimentos, nas condições em que cada gestor considera como ideal, quando há a solicitação de cortes de gastos. Nesse ponto há a possibilidade de o gestor entender as metas atribuídas a ele (como o corte de gastos) como ambíguas, pois não há um significado claro de como atingir esse objetivo.

Também, dois gestores apontaram que há reuniões semanais entre eles e a superintendência e, dentre outros assuntos discutidos, há a discussão do andamento do orçamento da instituição, mas somente conforme 
necessidade. Esses gestores participam da linha de hierarquia superior se considerar os entrevistados deste trabalho.

Os gestores clínicos específicos da Divisão de Enfermagem citaram a realização de reuniões internas do setor, em que há discussão do andamento orçamentário, juntamente com o planejamento de materiais que conta com a modificação ou manutenção dos itens. Isso pode ser verificado a seguir:

“(...)...a gente sempre senta durante o ano e discute para ver se tem alguma coisa sobrando que possa tirar para colocar outro (...)".

“(...) conversamos sobre isso, mas dentro do nosso grupo, que é o grupo de diretores, com a nossa diretora da enfermagem, a gente fala bastante sobre isso, aqui (planejamento de materiais) no meu setor na reunião com os enfermeiros, a reunião com os outros servidores a gente fala bastante no sentido de conscientizar como que acontece (...)."

Verifica-se que treze gestores citaram que seu planejamento é realizado ao final do ano para o período seguinte. Ao considerar as escolhas intertemporais dos gestores entrevistados, foi citado que elas podem ser influenciadas pelas ferramentas de gestão que a entidade utiliza (MERCHANT, 1990). Apesar de a entidade possuir o PPA, há um aparente foco das escolhas dos gestores no curto prazo, pois o planejamento do setor é efetuado somente para o período seguinte. Sendo assim, há uma aparente influência das ferramentas de gestão na tomada de decisão dos gestores entrevistados.

Somente um dos gestores apontou que a sua gestão é baseada em projetos de longo prazo, além da participação de planejamento de materiais citado pelos demais gestores. Esse gestor é responsável por uma relevante unidade na instituição e participa de projetos de longo prazo, como a ampliação da unidade que, conforme mencionado, pode se realizar em dez anos. $\mathrm{O}$ trecho em que foi identificado esse aspecto está mencionado abaixo.

\footnotetext{
"Você tem o orçamento que é aquele de longo prazo que aquele que é o que você pretende fazer dentro da instituição, para que isso ocorra a gente faz um projeto, estima custo, reforma de área física, vê a necessidade de pessoal, e pactua com a instituição a prioridade do que vai acontecer (...)."
}

Focando no andamento do orçamento, caso aconteça alguma atividade, procedimento ou consumo de recursos que esteja fora do orçamento do setor, ou que o extrapole, os gestores foram unânimes em citar que há a solicitação de complementação feita à administração que, (conforme análise) autoriza ou não. Quanto a isso foi observado que os gestores não veem conflitos nessa solicitação.

Outra questão é o consumo de materiais que determinado paciente pode necessitar e, conforme citado por outros gestores, isso é passado para a administração e essa "reposição" geralmente é aprovada.

Quando citada a consulta do orçamento para a tomada de decisão, dez gestores citaram que realizam essa consulta, principalmente no que tange à incorporação de novas tecnologias e ao planejamento do consumo para o próximo ano.

\footnotetext{
"Se incorpora ou não uma metodologia, na prática clínica além da questão legal dele ter sido aprovado para uso clínico, tem também a capacidade de pagar esse procedimento, sem dúvida nenhuma, inclusive como somos um setor muito dependente de tecnologia alta a gente tem sempre a pressão do custo presente. O limite é a própria disponibilidade de recursos."
}

Os gestores que citaram não consultar o orçamento para tomada de decisão são todos profissionais médicos. Ao analisar três gestores, desses cinco, verifica-se que são os mesmos gestores que citaram a falta do conhecimento, ou conhecimento de forma não aprofundada do orçamento, e que também não participam do planejamento de materiais do setor.

Os demais gestores que citaram não consultar o orçamento da instituição para tomada de decisão disseram que têm uma planilha eletrônica de acompanhamento do consumo de material do setor.

Pode-se verificar, portanto, que os gestores que têm a percepção de participar do processo orçamentário 
da instituição, consultam o orçamento para tomada de decisão. A literatura aponta que a participação no processo orçamentário é uma característica dessa ferramenta (LU, 2011), e que essa participação pode motivar o gestor a atingir as metas definidas, desde que também haja motivação (SEARFOSS; MONCZKA, 1973). Ressalta-se, também, que a literatura suporta a hipótese de que essa participação pode aumentar o desempenho dos hospitais (ABERNETHY; BROWNELL, 1999).

Ao discutir, com os entrevistados sobre a presença de metas orçamentárias, somente um dos gestores, que alegou desconhecimento do orçamento, argumentou não conhecer tais metas.

Dentre os demais gestores, cinco observaram como meta o limite de recursos imposto pelo centro de custo, ou seja, o teto de recursos que cada gestor tem. Além disso, três gestores identificaram, como metas atreladas ao orçamento, indicadores ligados à "produção", à "assistência", ao "número de exames", ou seja, indicadores ligados ao consumo dos recursos.

\subsection{Critérios para Tomada de Decisão}

Os gestores foram questionados quanto aos critérios utilizados para tomada de decisão. Os discursos dos entrevistados foram primeiramente submetidos a uma pré-análise que consistiu na organização dos discursos e identificação, dentro do roteiro semiestruturado, das questões que se referiam a Critérios de Tomada de Decisão.

Observa-se que os critérios relacionados ao tratamento do paciente ocorrem com maior frequência se os compararem aos demais. Entre os nove gestores que citaram o tratamento do paciente se destacam critérios como: risco para o paciente, questões clínicas e epidemiológicas, sofrimento do paciente, confiabilidade, aumento da segurança do paciente. Destes, seis são médicos, o que corrobora com autores (MARCH; OLSEN, 1989; NYLAND; PETTERSEN, 2004) que citam a possível orientação do corpo clínico guiado pela lógica da adequação das atitudes às necessidades.

Foi detectado que, possivelmente, na fase inicial da decisão o componente custo pode não ser levado em consideração no processo de tomada de decisão do gestor. Corrobora esse aspecto a incorporação de tecnologia realizada por determinado setor, que é por meio de um parecer, mas que não necessariamente inclui o componente impacto orçamentário.

Detectou-se também que pode haver dificuldades na instituição em levantar qual o real custo do procedimento, ou do paciente, pois não é uma informação que, a princípio, está clara. Ressalta-se que há uma complexidade em determinar os custos hospitalares, consequência da presença de uma diversidade de produtos (BONACIM; ARAUJO, 2010).

Esse componente "Custo", incluído na variável "Gastos", foi relacionado por cinco gestores. Além desse item, também foi citado "consumo de recursos", "estoque", "impacto orçamentário", "impacto no custo do protocolo clínico", "investimento".

Foi citado que a análise do impacto da utilização de um novo procedimento, ou da tomada de decisão, não tem que levar em conta somente o custo do item e, sim, do impacto no procedimento, ou protocolo, como um todo. Por exemplo, a tomada de decisão de aquisição de um medicamento, necessário para o tratamento do paciente, que tenha um custo mais elevado do que o utilizado, pode impactar o tempo de internação, que impacta no consumo de outros materiais. Sendo assim, há a aparente necessidade de analisar o impacto da tomada de decisão como um todo, e não especificamente em um determinado item. Esse tipo de decisão assemelha-se à tomada de decisão relacionada ao conceito de "farmacoeconomia". Verificou-se isso nessa passagem de um entrevistado, por exemplo:

“(...) ...nós fazemos pesquisa e nossas pesquisas não deixam de levar em consideração isso, o que é melhor em termos de resultados clínicos e é óbvio que na fase inicial esse componente despesa e custo ele não está embutido, mas é uma preocupação, mas a gente trabalha muito com evidência científica. Isso tem nível de evidência forte de que funciona nós vamos buscar a qualquer custo. A evidência científica não inclui somente o resultado, mas o custo também, não podemos ver somente o impacto na saúde, mas sim o impacto social disso, então é com essa linha de argumentos que discutimos com a administração.” 
Observa-se, também, que os gestores mais próximos à atividade fim do hospital, utilizam critérios voltados para o Tratamento do Paciente. Há uma aparente evidenciação de que quanto mais próximo o gestor se encontra da atividade fim do hospital, mais o foco volta-se para a assistência.

Pode-se ressaltar, portanto, com base nesses resultados, que há uma aparente tomada de decisão centralizada na melhoria de condições para o paciente. Esse achado demonstra um foco voltado para o paciente, como também demonstrado por Oyadomari et al. (2014). No entanto, o componente impacto orçamentário, mesmo com a aparente dificuldade em analisar o custo, não é deixado de lado, uma vez que os gestores citaram preocupação com esse aspecto, mas apontam que a análise precisa ser ampla, ou seja, a tomada de decisão precisa considerar o impacto no procedimento como um todo, e não somente no custo de determinado item.

Observa-se, também, que alguns gestores percebem a melhoria de condições e do tratamento do paciente com impacto direto no orçamento, pois a tentativa é de diminuir a permanência dele dentro do hospital, diminuindo assim o consumo de recursos por ele realizado. Ainda, há a percepção do impacto social que o procedimento pode trazer para o indivíduo.

\section{CONSIDERAÇÕES FINAIS}

O interesse inicial no tema apresentado se deu pela tentativa de contribuir com trabalhos que tratam da gestão em saúde, principalmente os que se referem a hospitais públicos. O possível diferencial deste trabalho é que são encontrados poucos trabalhos que discutem a relação orçamento e tomada de decisão em um hospital público. Além disso, com a identificação de variáveis presentes na tomada de decisão de gestores clínicos pode-se contribuir com a elaboração de um instrumento de coleta de dados capaz de contribuir com o diagnóstico da gestão em saúde.

As análises demonstram que há a interação dos gestores com o orçamento, no entanto, ela é aparentemente percebida, pela maioria que afirmou participar, como sendo predominantemente para planejamento de materiais do setor e incorporação de novas tecnologias. Portanto, em questão de planejamento de recursos, essa é a participação percebida pelos gestores no processo orçamentário.

Também foi percebido que o processo orçamentário pode impactar as escolhas intertemporais dos gestores, pois eles são exigidos, em sua maioria, a planejar as atividades e o consumo de recursos para, no máximo, o período seguinte. Portanto, há evidências que demonstram que o instrumento de gestão impacta esse tipo de escolha.

Ao comparar o discurso sobre a participação dos gestores clínicos na elaboração do orçamento com a possível consulta a esse processo para tomada de decisão, verificou-se que dez gestores consultam esse processo para tomada de decisão e, dentre os que citaram não consultar, todos possuem formação na área médica. Além disso, os que citaram não consultar são os mesmos que citaram não participar do planejamento de consumo do setor.

Dentre as variáveis citadas pelos gestores, as que foram percebidas por esse estudo como passíveis de se encontrar no processo orçamentário são: custo do material, acompanhamento do consumo de recursos (por meio do controle da assistência), impacto no protocolo clínico (considerando tanto o custo do item quanto o impacto no tratamento do paciente), investimento em atualização tecnológica, normatização (adequação às normas).

Esse trabalho tem o intuito de colaborar com a sistematização de um instrumento de coleta de dados utilizando as variáveis observadas neste estudo para investigar a relação dos gestores com o processo orçamentário de hospitais públicos que possuem similaridade à instituição estudada. Sendo assim, é sugestão de pesquisas futuras que esse instrumento seja sistematizado e que se investigue se essas variáveis também estão presentes em outras instituições de mesmo perfil.

\section{REFERÊNCIAS}

ABERNETHY, M. A.; BROWNELL, P. The role of budgets in organizations facing strategic change: an exploratory 
study. Accounting, Organizations and Society. v. 24, n. 3, p. 189-204, 1999.

ABERNETHY, M. A.; STOELWINDER, J. U. Budget use, task uncertainty, system goal orientation and subunit performance: a test of the - fitl hypothesis in not-for-profit hospitals. Accounting, OrganizationsandSociety.v. 16, n. 2, p. 105-120, 1991.

BARDIN, L.; Análise de Conteúdo (L. de A. Rego \& A. Pinheiro, Trads.). Lisboa: Edições 70. (Obra original publicada em 1977). 2011.

BONACIM, C. A. G.; ARAUJO, A. M. P. Gestão de custos aplicada a hospitais universitários públicos: a experiência do Hospital das Clínicas da Faculdade de Medicina de Ribeirão Preto da USP. Revista de Administração Pública. Rio de Janeiro, v. 44, n. 4, p. 903-931, 2010.

BITTAR, O. V.; MAGAlHÃES, A. Hospitais de ensino no estado de São Paulo: seis anos de acompanhamento. Revista de Administração em Saúde. v.12, p. 155-213, 2010. Número especial.

COOMBS, R. W. Accounting for the control of doctors: management information systems in hospitals. Accountin, OrganizationsandSociety. v. 12, n. 4, p. 389-404, 1987.

COSTA, P. S.; SILVA, E. P.; TIBÉRIO, C. S. B. Gestão estratégica de custos: estudo de caso aplicado a hospitais universitários. In: CONGRESSO INTERNACIONAL DE CUSTOS, 8., 2003. Anais... Uruguai.

DAL POZ, M. R., PIERANTONI, C. R., VARELLA, T. C. Produtividade e Desempenho dos Recursos Humanos nos Serviços de Saúde. Brasília: OPAS; 1997.

DALLORA, M. E. L. V.; FORSTER, A. C. A importância da gestão de custos em hospitais de ensino - considerações teóricas. Medicina (Ribeirao Preto. Online). v. 41, n. 2, p. 135-142, 2008.

ETHERINGTON, L.; TJOSVOLD, D. Managing Budget Conflicts: Contribution of Goal Interdependence and Interaction. Canadian Journal of Administrative Sciences. v. 15, n.2, p. 142-151, 1998.

FADEL, M. A .V.; FILHO, G. I .R. Percepção da qualidade em serviços públicos de saúde: um estudo de caso. Revista de Administração Pública. v. 43, n. 1. p. 7-22, 2009.

FREDERICK, S.; LOEWENSTEIN, G.; O’DONOGHUE, T. Time discounting and time preference: a critical review. Journal of Economic Literature. v. 40, n. 2, p. 351-401, 2002.

GLOUBERMAN, S.; MINTZBERG, H. Managing the care of health and curing the disease, Part I: Differentiation. Healthcare Management Review. v. 26, n. 1, p. 56-69, 1996.

GEORGESCU, I.; NARO, G. Budget pressures in hospitals: a qualitative study of RAPM among physicians. Comptabilité - Contrôle - Audit. v. 18, n. 3, p. 67-96, 2012.

HARTMANN, F. The Effects of Tolerance for Ambiguity and Uncertainty on the Appropriateness of Accounting Performance Measures. ABACUS. v. 41, n. 3, p. 241-264, 2005.

HOPWOOD, A. Accounting calculation and the shifting sphere of the economic. European Accounting Review. v. 1, n. 1, p. 125-143, 1992.

HOSPITAL DAS CLÍNICAS DA FACULDADE DE MEDICINA DE RIBEIRÃO PRETO (HCRP). Relatório de Atividades de 2013. 2014. Disponível em: <http://www.hcrp.fmrp.usp.br/sitehc/ upload\%5CRelatoriodeAtividades_HCRP_2013.pdf $>$ Acessado em: 27/09/2014.

KINNER, K.; PELLEGRINI, C. Expenditures for public health: assessing historical and prospective trends. American Journal of Public Health. v. 99, n. 10, p. 1780-1791, 2009.

KURUNMAKI, L. Professional vs financial capital in the field of health care-struggles for the redistribution of power and control. Accounting, Organizations and Society. v. 24, n. 2, p. 95-124, 1999.

LA FORGIA, G. M.; COUTTOLENC, B. F. Desempenho hospitalar no Brasil: em busca da excelência. São Paulo: Singular, 2009.

LAPSLEY, I. The accounting-clinical interface-implementing budgets for hospital doctors. ABACUS. v. 37, n. 1, p. 79-109, 2001.

LAVERTY, K. J. Economic "short-termism": the debate, the unresolved issues, and the implications for management practice and research. The Academy of Management Review.v. 21, n. 3, p. 825-860, 1996.

LIEBERMANN, Y.; UNGAR, M. Efficiency of consumer intertemporal choice under life cycle cost conditions. 
Journal of Economic Psychology. v. 23, n. 6, p. 729-748, 2002.

LU, C. T. Relationships among budgeting control system, budgetary perceptions, and performance: A study of public hospitals. African Journal of Business Management. v. 5, n. 15, p. 6261-6270, 2011.

MACINATI, M. S. NPM reforms and the perception of budget by hospital clinicians: lessons from two casestudies. Financial Accountability and Management. v. 26, n. 4, p. 422-442, 2010.

MARCH, J. G.; OLSEN, J. P. Rediscovering institutions: the organizational basis of politics. Hardcover. 1989.

MEDICI, A. C. Hospitais universitários: passado, presente e futuro. Rev. Assoc. Med. Bras., São Paulo, v. 47, n. 2, p. 149-156, 2001.

MERCHANT, K. A. Budgeting and the propensity to create budgetary slack.Accounting, Organizations and Society.v. 10, n. 2, p. 201-210, 1985.

MERCHANT, K. A. The effects of financial controls on data manipulation and management myopia. Accounting, Organizations and Society. v. 15, n. 4, p. 297-313, 1990.

NYLAND, K.; PETTERSEN, I. J. The control gap: the role of budgets, accounting information and (non-) decisions in hospital settings. Financial Accountability \& Management.v. 20, n. 1, p. 77-102, 2004.

NYLINDER, P. Perception of budgetary control: a study of differences across managers in Swedish public primary healthcare related to professional background and sex. Journal of Nursing Management. v. 19, n. 5, p. 664-672, 2011.

OUCHI, W. G. A conceptual framework for the design of organizational control mechanisms. Management Science. v. 25, n. 9, p. 833-848, 1979.

OYADOMARI, J. C. T. et al.. Uso do controle gerencial e decisões em organizações de saúde brasileiras: um estudo exploratório. BBR - Brazilian Business Review. v. 11, n. 2, p. 1-34, 2014.

PETTERSEN, I. J. Budgetary control of hospitals - ritual rhetorics and rationalized myths. Financial Accountability \& Management. v. 11, n. 3, p. 207-221, 1995.

PETTERSEN, I. J.; NYLAND, K. Management and controlof public hospitals - the use of performance measures in Norwegian hospitals.A case-study. The International Journal of Health Planning and Management. v. 21, n. 2, p. 133-149, 2006.

PRESTON, A. M.; COOPER, D. J.; COOMBS, R. W. Fabricating budgets: a study of the production of management budgeting in the national health service. Accounting, Organizations and Society. v. 17, n. 6, p. 561-593, 1992.

ROGOWSKI et al. Criteria for fairly allocating scarce health-care resources to genetic tests: which matter most? European Journal of Human Genetics. v. 22, n. 1, p. 25-31, 2014.

ROTTA, C. S. G. Utilização de indicadores de desempenho hospitalar como instrumento gerencial. Tese apresentada ao Departamento de Prática de Saúde Pública da Faculdade de saúde Pública da Universidade de São Paulo para obtenção do grau de doutorado.- Universidade de São Paulo, São Paulo, 2004.

SEARFOSS D. G.; MONCZKA, R. M. Perceived participation in the budget process and motivation to achieve the budget. Academy of Management Journal. v. 16, n. 4, p. 541-554, 1973.

VAN DER STEDE, W. A. The relationship between two consequences of budgetary controls: budget slack creation and managerial short-term orientation. Accounting, Organizations and Society, v. 25, n. 6, p. 609-622, 2000.

VOSS, C; TSIKRIKTSIS, N \& FROHLICH, M. Case research in operations management. International Journal of Operations \& Production Management, v. 22, n. 2, p. 195-219, 2002. 